\title{
Construction of the energy matrix for complex atoms Part I: General remarks
}

\author{
Magdalena Elantkowska ${ }^{1, a}$, Jarosław Ruczkowski², and Jerzy Dembczyński ${ }^{2}$ \\ 1 Laboratory of Quantum Engineering and Metrology, Faculty of Technical Physics, Poznań University of Technology, \\ Piotrowo 3, 60-965 Poznań, Poland \\ 2 Institute of Control and Information Engineering, Faculty of Electrical Engineering, Poznań University of Technology, \\ Piotrowo 3A, 60-965 Poznań, Poland
}

Received: 4 November 2014 / Revised: 12 December 2014

Published online: 29 January 2015

(C) The Author(s) 2015. This article is published with open access at Springerlink.com

\begin{abstract}
We present a method, which allows to analyse complex electronic systems composed of configurations including up to four open shells. The perturbative treatment, taking into account all electromagnetic interactions expected in the atom up to second-order perturbation theory, is described.
\end{abstract}

\section{Introduction}

The foundation of the description of an atomic structure is the assumption that the observed transition energy $h \nu$ is the difference between two electronic energy levels, or two hyperfine structure sublevels in an atom. It should be clearly emphasised that the value of energy levels of fine structure $(f s)$ or hyperfine structure $(h f s)$ sublevels is the sum of all possible electromagnetic interactions appearing in an atom. We will try to show how, with high precision, it is possible to determine quantitatively contributions of particular interactions and give an exact definition of the evaluated parameters describing the interactions in the atom.

The way to obtain information regarding the structure of an atom, mechanisms of interaction between the electrons, and the interactions of electron shell with the nucleus is to find the most accurate wave function describing the state of an atom. The knowledge of the exact wave function allows to specify, according to the quantum mechanics rules, the expected values of observables, which are the attributes of the atomic structure that are measurable, thus making their experimental verification possible. Such attributes are, for example, energy levels of an atom, lifetimes of an atom in the excited states, as well as the energy sublevels of the hyperfine structure, where the nucleus of an atom has an angular momentum $I>0$ and the calculations are performed on the basis of $|v S L J F\rangle$.

We developed a method, which allows to analyze a complex electronic system composed of a configuration of up to four open shells, taking into account all electromagnetic interactions expected in an atom, in accordance with the second-order perturbation theory. Within this theory, all possible combinations following the excitation of one or two electrons from closed shells to particular open shells were considered. The appropriate formulae and computer codes have been developed for many years by our research group.

Recently, we have presented a method for determining oscillator strengths that is an alternative to the commonly used, purely theoretical calculations, or to the semi-empirical approach combined with theoretically calculated transition integrals [1-3]. The angular coefficients of the transition matrix in pure $S L$ coupling were found from straightforward Racah algebra. The transition matrix was transformed into the actual intermediate coupling by the fine structure eigenvectors obtained from the semi-empirical approach. The transition integrals were treated as free parameters in least squares fits to experimental $g f$ values. As an example, the results of the calculation for the electric dipole transitions for Sc II, Ti II and Nb II were presented.

Satisfactory results obtained in these works were only possible due to the very precise wave functions obtained from the semi-empirical multi-configuration fine structure analysis, taking into account the first- and second-order effects of perturbation theory. Therefore, we have decided to present explicitly the formulae developed by us over many years.

\footnotetext{
a e-mail: magdalena.elantkowska@put.poznan.pl
} 


\section{Construction of the fine structure energy matrix}

The construction of the energy matrix of the Hamiltonian [4-7] describing the fine structure of an atom requires calculation of numerous integrals dependent on the angular coordinates and various radial integrals. The integrals over angular coordinates can be exactly determined, which is not possible in the case of radial integrals. Therefore, the matrix elements of the Hamiltonian are considered as linear combinations of radial integrals where the angular integrals serve as the coefficients of expansions. The radial integrals are treated as free (or constrained) parameters, which can be determined by fitting the calculated levels to the experimental ones with the least squares method. It is very important to clearly define the radial integral parameters, since their starting values for the iterative $f s$ LSQ-fit procedure can be taken from $a b$ initio calculations, and in view of their physical interpretation.

In the construction of an energy matrix we took into account all electromagnetic interactions occurring in the atom.

\subsection{First-order effects of perturbation theory}

All the operators of which the fine structure Hamiltonian is composed can be written as one- or two-particle operators. The method for calculating their matrix elements is generally described in Cowan's book [8] and Donlan's paper [9]. The calculation details of the matrix elements of the particular Hamiltonian constituents are discussed below.

In our computer procedure the first-order matrix elements are calculated as follows:

$$
\left\langle\psi|\mathbf{H}| \psi^{\prime}\right\rangle=\left\langle\psi\left|\sum_{i} \mathbf{H}_{i}\right| \psi^{\prime}\right\rangle=(\text { angular part of operator }) \times(\text { radial part }),
$$

where $\mathbf{H}_{i}$ represents a particular interaction which we include in our $f s$ energy matrix; $\psi, \psi^{\prime}$ represent particular states of the considered system configurations.

\subsubsection{Electrostatic interaction}

$\mathbf{H}_{\mathbf{1}}$ - the two-body operator of electrostatic interaction (usually referred to as $\mathbf{G}$ ).

- Intraconfiguration matrix elements. The formulae allowing to calculate the matrix elements of the Coulomb interaction were given by Donlan [9], Wybourne [10,11] and Roth [12].

- Interconfiguration matrix elements. The rules of calculating the interconfiguration matrix elements of the Coulomb interaction are also enclosed in Cowan's book [8]; however, we have used the more convenient coupling of open shells and the $S L$-coupling, traditionally preferred in the $h f s$ analysis, instead of the convention prevailing in [9], where successive shells are coupled in the $L S$-scheme. All formulae were described in our earlier works $[13,14]$ and were derived in this work.

\subsubsection{Magnetic interaction}

- Spin-orbit interaction. $\mathbf{H}_{\mathbf{2}}$ - the one-body operator of spin-orbit interaction (then determined as $\mathbf{H}_{\mathrm{so}}$ ). The formulae for the matrix elements of spin-orbit interaction can be found in Wybourne's monograph [11], in our works $[15,16]$ and were also derived within this work. The radial factor of the spin-orbit operator $\mathbf{H}_{\text {so }}$ is commonly referred to as the spin-orbit parameter $\zeta_{\mathrm{nl}}$.

- Mixed magnetic interactions acting in core $(n l)^{N} \cdot \mathbf{H}_{\mathbf{3}}, \mathbf{H}_{\mathbf{4}}$ and $\mathbf{H}_{\mathbf{5}}$ - the two-body operators of orbit-orbit, spinother orbit and spin-spin interactions, respectively (described in the literature as $\mathbf{H}_{\mathrm{oo}}, \mathbf{H}_{\mathrm{soo}}, \mathbf{H}_{\mathrm{ss}}$ ). The completeness of $f_{s}$ analysis requires including magnetic interactions of the kinds: orbit-orbit, spin-other orbit and spin-spin interactions. The forms of magnetic interaction operators were published in the papers $[4,6,7,17]$. The matrix elements for configurations $\mathrm{d}^{N}$ were given by Judd [18] and Barnes [19]. The accurate formulae, allowing to calculate the matrix elements of these operators in core $(n l)^{N}$ were presented in our work [13]. The radial parameters are denoted as $M^{k}$.

- Mixed magnetic interactions acting between electrons $l-l^{\prime} . \mathbf{H}_{\mathbf{6}}$ and $\mathbf{H}_{\mathbf{7}}$ - the two-body operators of spin-other orbit and spin-spin interactions, respectively (described in the literature [20,21] as $\mathbf{H}_{\mathbf{1 2}}^{\text {soo }}, \mathbf{H}_{\mathbf{1 2}}^{\mathrm{ss}}$ ). In our work [13] the matrix elements for the so called mixed magnetic interactions were calculated irrespectively of the term responsible for the magnetic interaction between the core and the added electrons. Following the method described by Rudzikas [20,21], the expressions for two-electron reduced matrix elements of spin-orbit and spin-spin interaction between electrons $l-l_{1}, l-l_{2}, l_{1}-l_{2}$ were derived and included into the energy matrix. The radial parameters are denoted as $M^{k}$, $N^{k}, K^{k}$. 


\subsection{Second-order effects of perturbation theory}

The matrix elements of the second-order perturbations were calculated taking into account the corrections related to the operators $\mathbf{H}_{1}$ and $\mathbf{H}_{\mathbf{2}}$.

\subsubsection{Effective electrostatic interactions}

As shown by Trees [22], Rajnak and Wybourne [23,24], Judd [25], Racah and Stein [26] and Feneuille [27-30], an inclusion of configuration interactions (electrostatic coupling with distant configurations) with the use of second-order perturbation theory essentially improves the agreement between experimental and calculated values of energy levels. These effects are represented by two- and three-body parameters. The Hamiltonian describing the fine structure of an atom was written by Armstrong and Feneuille [6,7] in the form of the effective operators acting on nonrelativistic wave function. In this work our energy matrix is extended by the elements taking into account electrostatic coupling and electrostatically correlated spin-orbit coupling between the configurations of the system considered and distant configurations. Generally, for the configurations containing up to three open electronic shells, these matrix elements originate from the second-order perturbation theory and can be schematically expressed as follows:

$$
-\sum_{\psi_{1} \neq \psi, \psi^{\prime}}\left[\left\langle\psi|\mathbf{G}| \psi_{1}\right\rangle \times\left\langle\psi_{1}|\mathbf{G}| \psi^{\prime}\right\rangle\right] / \Delta E=-(\text { angular part }) \times(\text { radial part }),
$$

where

$$
\begin{aligned}
\psi & =\left(n_{0} l_{0}\right)^{4 l_{0}+2}{ }^{1} S,\left(n_{1} l_{1}\right)^{N_{1}} S_{1} L_{1},\left(\left(n_{2} l_{2}\right)^{N_{2}} S_{2} L_{2},\left(n_{3} l_{3}\right)^{N_{3}} S_{3} L_{3}\right) S_{4} L_{4} ; S L, \\
\psi^{\prime} & =\left(n_{0} l_{0}\right)^{4 l_{0}+2}{ }^{1} S,\left(n_{1} l_{1}\right)^{N_{1}} S_{1}^{\prime} L_{1}^{\prime},\left(\left(n_{2} l_{2}\right)^{N_{2}} S_{2}^{\prime} L_{2}^{\prime},\left(n_{3} l_{3}\right)^{N_{3}} S_{3}^{\prime} L_{3}^{\prime}\right) S_{4}^{\prime} L_{4}^{\prime} ; S L,
\end{aligned}
$$

and $\psi_{1}$ denote all perturbing virtual states taken into account in our system, resulting from the excitation of one or two electrons from a closed $\left(n_{0} l_{0}\right)^{4 l_{0}+2}$ shell into the partially filled $(n l)^{N}$ shells, for example,

$$
\psi_{1}=\left(n_{0} l_{0}\right)^{4 l_{0}} S_{0} L_{0},\left(n_{1} l_{1}\right)^{N_{1}+2} S_{1}^{\prime \prime} L_{1}^{\prime \prime},\left(\left(n_{2} l_{2}\right)^{N_{2}} S_{2}^{\prime \prime} L_{2}^{\prime \prime},\left(n_{3} l_{3}\right)^{N_{3}} S_{3}^{\prime \prime} L_{3}^{\prime \prime}\right) S_{4}^{\prime \prime} L_{4}^{\prime \prime} ; S L .
$$

The angular coefficients are the result of the coupling of angular momenta of the operator G. Radial parameters have denotations which code the interacting configurations and specify the interactions. Denotations of particular radial parameters were described in [31].

In our procedure excitations of one or two electrons from a closed shell to all open shells are considered under the following conditions:

$$
\left|l_{i}-l_{0}\right|=0,2 \quad \text { and } \quad N_{1}+N_{2}+N_{3}=N_{1}^{\prime}+N_{2}^{\prime}+N_{2}^{\prime}
$$

\subsubsection{Electrostatically correlated spin-orbit interactions}

The contributions from the second-order perturbation theory to electrostatically correlated spin-orbit interactions (CSO) are defined as follows:

$$
-\sum_{\psi_{1} \neq \psi, \psi^{\prime}}\left[\left\langle\psi|\mathbf{G}| \psi_{1}\right\rangle \times\left\langle\psi_{1}\left|\mathbf{H}_{\mathrm{so}}\right| \psi^{\prime}\right\rangle+\left\langle\psi\left|\mathbf{H}_{\mathrm{so}}\right| \psi_{1}\right\rangle \times\left\langle\psi_{1}|\mathbf{G}| \psi^{\prime}\right\rangle\right] / \Delta E=-(\text { angular part }) \times(\text { radial part }),
$$

where

$$
\begin{aligned}
\psi & =\left(n_{0} l_{0}\right)^{4 l_{0}+2}{ }^{1} S,\left(n_{1} l_{1}\right)^{N_{1}} S_{1} L_{1},\left(\left(n_{2} l_{2}\right)^{N_{2}} S_{2} L_{2},\left(n_{3} l_{3}\right)^{N_{3}} S_{3} L_{3}\right) S_{4} L_{4} ; S L J \\
\psi^{\prime} & =\left(n_{0} l_{0}\right)^{4 l_{0}+2}{ }^{1} S,\left(n_{1} l_{1}\right)^{N_{1}} S_{1}^{\prime} L_{1}^{\prime},\left(\left(n_{2} l_{2}\right)^{N_{2}} S_{2}^{\prime} L_{2}^{\prime},\left(n_{3} l_{3}\right)^{N_{3}} S_{3}^{\prime} L_{3}^{\prime}\right) S_{4}^{\prime} L_{4}^{\prime} ; S^{\prime} L^{\prime} J
\end{aligned}
$$

and $\psi_{1}$ denote all perturbing virtual states, resulting from the excitation of an electron from a closed $\left(n_{0} l_{0}\right)^{4 l_{0}+2}$ shell into the partially filled $(n l)^{N}$ shells, for example,

$$
\psi_{1}=\left(n_{0} l_{0}\right)^{4 l_{0}+12} L_{0},\left(n_{1} l_{1}\right)^{N_{1}+1} S_{1}^{\prime \prime} L_{1}^{\prime \prime},\left(\left(n_{2} l_{2}\right)^{N_{2}} S_{2}^{\prime \prime} L_{2}^{\prime \prime},\left(n_{3} l_{3}\right)^{N_{3}} S_{3}^{\prime \prime} L_{3}^{\prime \prime}\right) S_{4}^{\prime \prime} L_{4}^{\prime \prime} ; S^{\prime \prime} L^{\prime \prime} J
$$

In these cases the angular coefficients also result from the angular parts of the operators: the two-body electrostatic interaction operator $\mathbf{G}$ and the one-body spin-orbit operator $\mathbf{H}_{\text {so }}$. Denotations of particular radial parameters were also described in [31]. 


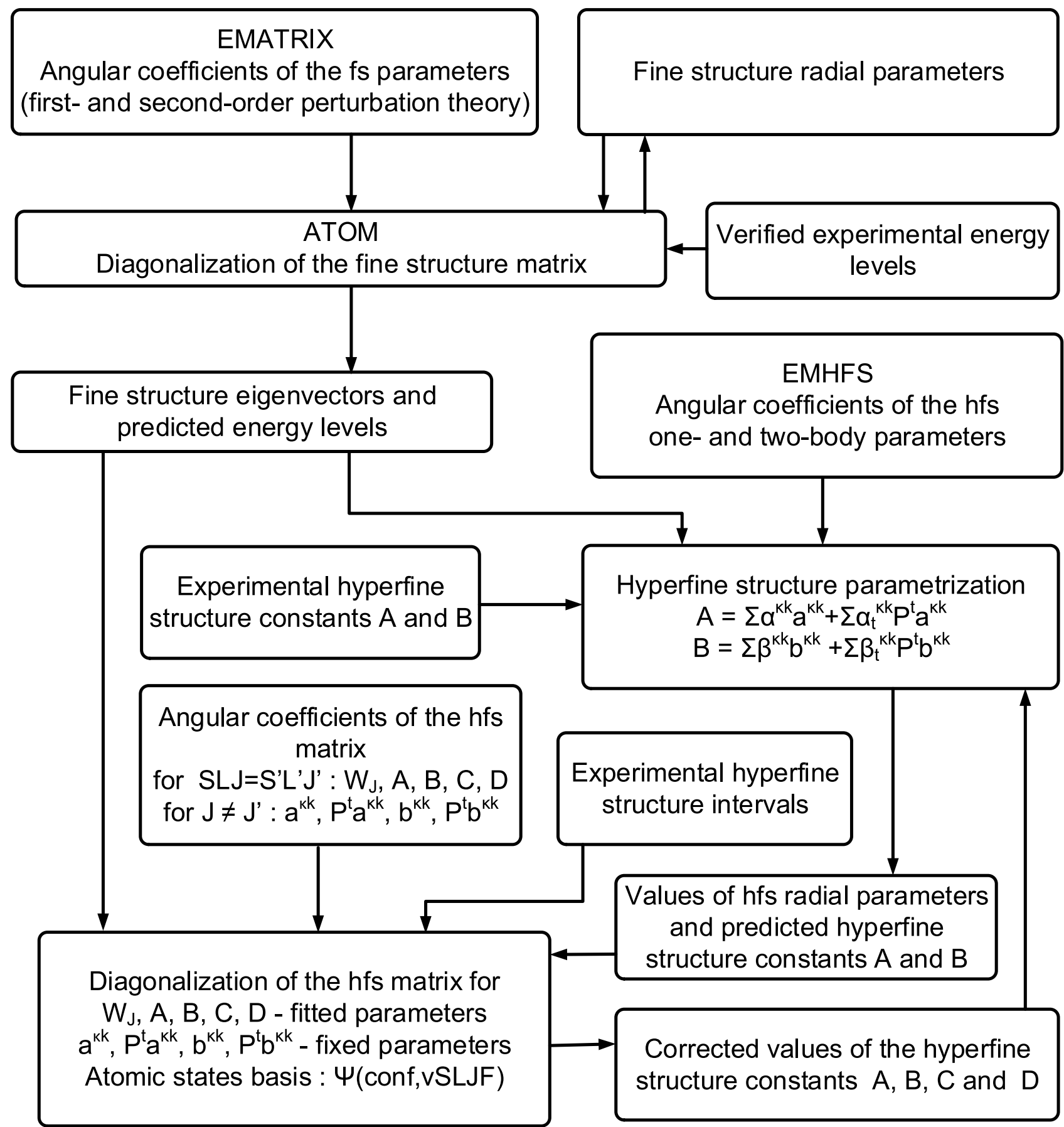

Fig. 1. Scheme of the procedures for the fine and hyperfine structure analysis.

In the case of CSO the following condition has to be fulfilled:

$$
\left|l_{0}-l_{1}\right|=0 \quad \text { and } \quad \kappa k=11, K=0 .
$$

The matrix elements determined from relations (2) and (6) under conditions (5) and (9) were included in the fine structure energy matrix (fig. 1).

Generally, the matrix elements for configurations containing up to four open electronic shells $\left(\left(n_{1} l_{1}\right)^{N_{1}}\left(n_{2} l_{2}\right)^{N_{2}}\left(n_{3} l_{3}\right)^{N_{4}}\left(n_{4} l_{4}\right)^{N_{4}}\right)$ originate from the first-order effects of perturbation theory, and the matrix elements for configurations containing up to three open shells originate from the second-order perturbation theory, which means that we inferred all formulae describing the above-mentioned interactions. The work to calculate matrix elements of various operators, particularly for complex configurations with several open shells was very 
time-consuming, because it required calculating numerous sums over quantum numbers of the momentum type and their projections, leading to the occurrence of the so-called $3 n j$ - and $j m$-coefficients (in this work up to $15 j$-coefficients). We used the work of Jucys and co-workers [32], where the sum rules on $j m$-coefficients were given. Due to the large number of formulae, they will be published explicitly as separate works.

\section{Construction of the hyperfine structure energy matrix}

In the construction of the hyperfine structure energy matrix we used the operator

$$
\mathbf{H}_{\mathrm{hfs}}=\sum_{K=1}^{3} \mathbf{T}_{e}^{(\kappa k) K} \cdot \mathbf{T}_{n}^{(K)}
$$

provided by Schwartz [33,34], where $\mathbf{T}_{e}^{(\kappa k) K}$ and $\mathbf{T}_{n}^{(K)}$ denote the electron and the nuclear operator, respectively.

For the operator $\mathbf{T}_{n}^{(K)}$ we used definitions formulated by Lindgren and Rosen [17], and for the operator $\mathbf{T}_{e}^{(\kappa k) K}$ the well-known formula introduced by Sandars and Beck [35], i.e.

$$
\begin{aligned}
& \mathbf{T}_{e}^{(\kappa k) 1}=\frac{\mu_{0} \mu_{B}}{2 \pi} \sum_{i=1}^{N}\left[\mathbf{l}_{i}\left\langle r^{-3}\right\rangle^{01}-\sqrt{10}\left(\mathbf{s}_{i} \mathbf{C}_{i}^{2}\right)^{(1)}\left\langle r^{-3}\right\rangle^{12}+\mathbf{s}_{i}\left\langle r^{-3}\right\rangle^{10}\right] \\
& \mathbf{T}_{e}^{(\kappa k) 2}=\frac{e}{4 \pi \epsilon_{0}} \sum_{i=1}^{N}\left[-\mathbf{C}_{i}^{2}\left\langle r^{-3}\right\rangle^{02}+\sqrt{\frac{3}{10}}\left(\mathbf{U}_{i}^{(13) 2}\left\langle r^{-3}\right\rangle^{13}+\mathbf{U}_{i}^{(11) 2}\left\langle r^{-3}\right\rangle^{11}\right)\right] \\
& \mathbf{T}_{e}^{(\kappa k) 3}=\frac{\mu_{0} \mu_{B}}{2 \pi} \sum_{i=1}^{N}\left[\sqrt{\frac{5}{3}}\left(\mathbf{C}_{i}^{2} \mathbf{l}_{i}\right)^{3}\left\langle r^{-5}\right\rangle^{03}-6\left(\mathbf{s}_{i} \mathbf{C}_{i}^{4}\right)^{(3)}\left\langle r^{-5}\right\rangle^{14}+\sqrt{\frac{5}{3}}\left(\mathbf{s}_{i} \mathbf{C}_{i}^{2}\right)^{(3)}\left\langle r^{-5}\right\rangle^{12}\right] \\
& \mathbf{T}_{e}^{(\kappa k) 4}=\frac{e}{4 \pi \epsilon_{0}} \sum_{i=1}^{N}\left[-\mathbf{C}_{i}^{4}\left\langle r^{-5}\right\rangle^{04}+\sqrt{\frac{1}{6}}\left(\mathbf{U}_{i}^{(15) 4}\left\langle r^{-5}\right\rangle^{15}+\mathbf{U}_{i}^{(13) 4}\left\langle r^{-5}\right\rangle^{13}\right)\right] .
\end{aligned}
$$

For atoms with many electrons in an unclosed shell the magnetic dipole and electric quadrupole hyperfine interactions are normally analysed using the one-electron radial parameters defined as

$$
\begin{aligned}
a_{n l}^{\kappa k} & =\frac{\mu_{0}}{4 \pi} \frac{2 \mu_{B}}{h} \frac{\mu_{I}}{I}\left\langle r^{-3}\right\rangle_{n l}^{\kappa k} & \kappa k=01,12, \\
a_{n l}^{\kappa k} & =\frac{\mu_{0}}{4 \pi} \frac{2 \mu_{B}}{h} \frac{\mu_{I}}{I} \frac{2}{3}\left\langle r^{-3}\right\rangle_{n l}^{\kappa k} & \kappa k=10, \\
b_{n l}^{\kappa k} & =\frac{1}{4 \pi \epsilon_{0}} \frac{e^{2}}{h} Q\left\langle r^{-3}\right\rangle_{n l}^{\kappa k} & \kappa k=02,13,11,
\end{aligned}
$$

where $a^{01}$ represents the interaction between the nuclear magnetic-dipole moment $\mu_{I}$ and the magnetic field produced by purely orbital motion of the electrons, $a^{12}$ represents the interaction between $\mu_{I}$ and the magnetic moment of the electron, $a^{10}$ represents the relativistic and configuration interaction (core polarization), $b^{02}$ represents the electrostatic interaction between a nucleus and electron configurations, that have a spherically symmetric charge distribution which is finite at the nucleus, and $b^{13}, b^{11}$ are purely relativistic in nature.

In the first-order effects of perturbation theory, it was possible to calculate the matrix elements up to four open shells. The one-electron radial parameters $a^{\kappa k}, b^{\kappa k}, c^{\kappa k}$ and $d^{\kappa k}$ have the same definitions for all $n l$ electrons in the even and odd systems.

The second-order $h f s$ matrix elements are formed in the same manner as for $f s$ energy matrix and spin-orbit operator is replaced by $h f s$ operator.

Contributions from the second order perturbation theory to electrostatically correlated hyperfine interactions (CHFS) are defined as follows:

$$
-\sum_{\psi_{1} \neq \psi, \psi^{\prime}}\left[\left\langle\psi|\mathbf{G}| \psi_{1}\right\rangle \times\left\langle\psi_{1}\left|\mathbf{T}^{(\kappa k) K}\right| \psi^{\prime}\right\rangle+\left\langle\psi\left|\mathbf{T}^{(\kappa k) K}\right| \psi_{1}\right\rangle \times\left\langle\psi_{1}|\mathbf{G}| \psi^{\prime}\right\rangle\right] / \Delta E=-(\text { angular part) } \times(\text { radial part }),
$$

where $\psi, \psi_{1}$ and $\psi^{\prime}$ are defined in eq. (7).

In these cases also the angular coefficients result from the angular parts of the operators: a two-body electrostatic interaction operator $\mathbf{G}$ and a one-body operator $\mathbf{T}^{(\kappa k) K}$; the latter may represent a hyperfine $\left(H_{\mathrm{hfs}}\right)$ interaction. 
Denotations of particular $h f s$ radial parameters $\left(P^{t}\left(n_{i} l_{i} n_{0} l_{0}, n_{i} l_{i} n_{i}^{\prime} l_{i}^{\prime}\right) P^{\kappa k}\left(n_{0} l_{0}, n_{i} l_{i}\right)\right)$ and interpretation of calculated $A$ and $B$ constants expressed as a linear combination one- and two-body contributions were described in detail in [31].

In the case of CHFS the following relations hold:

- for magnetic dipole interactions $K=1$,

$$
\left|l_{0}-l_{1}\right|=0, \quad \text { if } \quad \kappa k=01,10 \quad \text { and } \quad\left|l_{0}-l_{1}\right|=0,2, \quad \text { if } \quad \kappa k=12 ;
$$

- and for electric quadrupole interactions $K=2$,

$$
\left|l_{0}-l_{1}\right|=0,2, \quad \text { if } \quad \kappa k=02 \quad \text { and } \quad\left|l_{0}-l_{1}\right|=0, \quad \text { if } \quad \kappa k=11,13 .
$$

The derived formulae describing the interaction described above will be presented explicitly in separate works.

\section{Parameterization history of fine and hyperfine structure of the atom}

The analysis of the fine structure, which takes into account only the first-order effects, such as the electrostatic interactions, represented by Slater integrals, began with the classic paper of Slater [36] and spin-orbit interaction ( $\zeta$ parameter) $[37,38]$, is for most cases insufficient. The extensive list of the $f s$-analyses carried out by the $f s$ LSQfit method was given by Judd in his review paper [39]. The method of parametrization of interactions in the fine structure for complex atoms in extended model space of $\left(n \mathrm{~d}+n^{\prime} \mathrm{s}\right)^{N+2}+\sum_{i, j} n \mathrm{~d}^{N+2-w_{i}-w_{j}} n_{i} l_{i}^{w_{i}} n_{j} l_{j}^{w_{j}}$ configurations was presented in our paper from 1996 [13], which also included corrections of errors from earlier papers of other authors $[24,40]$.

The monograph of Rudzikas Theoretical Atomic Spectroscopy presents a complete up-to-date guide to the theory of modern spectroscopy of atoms. It describes contemporary state of the theory of many-electron atoms and ions and the peculiarities of their structure and spectra.

The scope of the present paper concerns the complex atom fine structure for the configurations containing up to four open electronic shells $(n l)^{N}\left(n_{1} l_{1}\right)^{N_{1}}\left(n_{2} l_{2}\right)^{N_{2}}\left(n_{3} l_{3}\right)^{N_{3}}$ taking into account both the first-order effects of perturbation theory (electrostatic, spin-orbit and mixed magnetic interactions) and the second-order effects of the perturbation theory (effective electrostatic interactions and electrostatically correlated spin-orbit interactions).

The correctness of the fine structure wave functions was verified by applying the main quantitative results of the $f_{s}$ LSQ-fit to the calculation of other properties of the atom, in the present case the hyperfine structure splitting and the oscillator strengths for electric dipole transitions.

The development of the hyperfine structure $(h f s)$ theory was initiated with the classic paper of Sandars and Back [35]. They developed a theory which simplifies the calculation and interpretation of relativistic $h f s$ effects in many-electron atoms. This theory leads to three effective radial integrals for each open shell and for each multipole interaction. As shown by Judd [41,42] and Sandars [43], the configuration interaction (CI) effects caused by excitations from closed shells to empty shells can be included in an effective Hamiltonian of the same form. This theory was applied by Lindgren and Rosen [17] to analyse a large number of experimental ( $h f s$ ) data mainly in atomic ground configurations, and by Büttgenbach [44] to analyse $h f s$ in $4 \mathrm{~d}$ - and $5 \mathrm{~d}$-shell atoms. In these analyses of $h f s$ data the effective radial integrals were treated as free parameters which were fitted to the experimental results in order to consider some relativistic $C I$ effects. The experimental $h f s$ radial parameters obtained in this way show a rather poor agreement with theoretical results of relativistic Hartree-Fock calculations $[17,44,45]$ It demonstrates, that more contributions exist to the $h f s$ splittings which should be taken into account. Bauche-Arnoult [46,47], Armstrong [4] as well as Lindgren and Morrison [5] showed that configuration effects for the second-order perturbation theory can be split into to parts: the first (one-body) part, which is common to all terms of a given configuration, and the second (two-body) part depending on the $S L$-terms studied. Bauche-Arnould [46,47] defined the effective operators, which described the crossed second-order effects of electrostatic and hyperfine interactions, for all types of excitations appearing in the configurations $l^{N}$ or $l^{N} l^{\prime}$. Assuming pure $S L$-coupling, Bauche-Arnoult found regularities in the contributions from electrostatically correlated second-order $h f s$ to the different effective radial parameters. Later, in 1985, Dembczyński [48] elaborated a new parametrization method which took into account simultaneously one- and two-body interactions in atomic $h f s$ structure of the configurations $(3 \mathrm{~d}+4 \mathrm{~s})^{N+2}$. This method was applied to the interpretation of iron, vanadium, titanium and cobalt atoms [49-52]. In the next years the above mentioned method of parametrization of interactions in the hfs was extended up to three open electronic shells [13,16]. Remarks on the interpretation of very high-precision measurements of hyperfine structure splittings in neutral and singly ionised complex atoms were presented in our work [53,54].

Recently, we have presented a critical analysis of the methods of interpretation of the hyperfine structure of free atoms and ions [31]. The method of quantitative determination of two-body contributions to the hyperfine structure, resulting from the excitations from electronic closed shells to open shells and from open shells to empty shells, was described therein. Our earlier method [48] was developed for the system $(3 \mathrm{~d}+4 \mathrm{~s})^{N}$, and thus excitations of the kind 
"closed $n_{0} \mathrm{~d}$ shell - open $n \mathrm{~d}$ shell" did not occur. As an example we considered the model space $(5 \mathrm{~d}+6 \mathrm{~s})^{3}$ of the lanthanum atom.

Another problem which can cause discrepancies in some cases is the method of determination of the hyperfine structure constants $A, B, C$ and $D$ from the measured hyperfine intervals. A simplified description of the "repulsion" effect for the hyperfine structure sublevels with the same values of quantum number $F$ yields a substantial distortion of the information concerning quadrupole and higher-order interactions. Is was first noticed by Casimir [55], and referred to as "pseudoquadrupole" or "pseudooctupole" interactions. Therefore, in order to guarantee the correctness of determination of the hyperfine structure constants $A, B, C$ and $D$ in our approach, a segment called "diagonalization of the hyperfine structure energy matrix" within the basis of states $\Psi$ (configuration, $v S L J F$ ) was introduced [56], as described below. In this way we eliminate one of the possible sources of discrepancies.

The procedures of analysis of experimental data concerning the structure of complex atoms, developed by our group, are schematically outlined in fig. 1.

\section{Concluding remarks}

The observed values of the electromagnetic interactions in the atom are the sum of well-defined contributions, described in the first- and second-order perturbation theory. The radial parameters, of both the first and second order, are in our semi-empirical computer procedures determined in an iterative way on the basis of experimental data. This ensures that an increasing number of experimental data (e.g. new energy levels), provide quantitative determination for more and more contributions to the observed energy values, and consequently improve the accuracy of the obtained wave functions in the iterative procedures. Hereby, the difference between the measured values and the values calculated from eigenfunctions will tend to zero.

A full description of the second-order effects require the derivation and programming of several hundred formulae based on the addition of the quantum angular momentum theory (Racah algebra). It should be noted that in the construction of our energy matrix, there were no simplifications neglecting certain type of interactions, in the belief that they could be insignificant.

The implementation and dissemination of computer procedures will accelerate the processing of the experimental databases and result in a continuous increase in the number of known electronic levels, which will allow to determine quantitatively all the electromagnetic interactions provided by the theory of atomic structure.

Our intention is to publish in separate papers an explicit form of the derived formulae, describing the second-order interaction for the different types of configurations.

ME thanks the Poznań University of Technology for financial support within the project 06/63/DSPB/0314. JD and JR thank the Poznań University of Technology for financial support within the project 04/45/DSPB/0121.

Open Access This is an open access article distributed under the terms of the Creative Commons Attribution License (http://creativecommons.org/licenses/by/4.0), which permits unrestricted use, distribution, and reproduction in any medium, provided the original work is properly cited.

\section{References}

1. J. Ruczkowski, M. Elantkowska, J. Dembczyński, J. Quant. Spectrosc. Radiat. Transf. 145, 20 (2014) http://dx.doi.org/10.1016/j.jqsrt.2014.04.018.

2. J. Ruczkowski, M. Elantkowska, J. Dembczyński, J. Quant. Spectrosc. Radiat. Transf. 149, 168 (2014) http://dx.doi.org/10.1016/j.jqsrt.2014.08.010.

3. J. Ruczkowski, S. Bouazza, M. Elantkowska, J. Dembczyński, J. Quant. Spectrosc. Radiat. Transf. 155, 1 (2015) http://dx.doi.org/10.1016/j.jqsrt.2014.12.014.

4. L. Armstrong Jr., Theory of the Hyperfine Structure of Free Atoms (Willey-Interscience, New York, 1971).

5. I. Lindgren, J. Morrison, Atomic Many-Body Theory (Springer-Verlag, Berlin, Heidelberg, New York, 1982).

6. L. Armstrong Jr., S. Feneuille, Phys. Rev. 173, 58 (1968) http://dx.doi.org/10.1103/PhysRev.173.58.

7. L. Armstrong Jr., S. Feneuille, Adv. At. Mol. Phys. 10, 1 (1974) http://dx.doi.org/10.1016/S0065-2199 (08) 60346-2.

8. R.D. Cowan, The Theory of Atomic Structure and Spectra (Berkeley University of California Press, Berkeley, 1981).

9. V.L. Donlan, J. Chem. Phys. 52, 3431 (1970) http://dx.doi.org/10.1063/1.1673507.

10. B.G. Wybourne, J. Math. Phys. 4, 354 (1963) http://dx.doi.org/10.1063/1.1703961.

11. B.G. Wybourne, Spectroscopic properties of Rare Earths (Interscience, New York, 1965).

12. Ch. Roth, Can. J. Spect. 26, 25 (1981).

13. J. Dembczyński, G. Szawioła, M. Elantkowska, E. Stachowska, J. Ruczkowski, Phys. Scr. 54, 444 (1996) http://dx.doi.org/10.1088/0031-8949/54/5/004. 
14. M. Elantkowska, J. Ruczkowski, J. Dembczyński, Phys. Scr. 59, 49 (1999) http://dx.doi.org/10.1238/Physica.Regular.059a00049.

15. J. Dembczyński, Physica C 100, 105 (1980) http://dx.doi.org/10.1016/0378-4363(80)90067-4.

16. J. Dembczyński, M. Elantkowska, K. Bekk, H. Rebel, M. Wilson, Z. Phys. D 13, 181 (1989) http://dx.doi.org/10.1007/BF01436955.

17. I. Lindgren, A. Rosen, Case Studies in Atomic Physics, Vol. 4 (1974).

18. B.R. Judd, H.M. Crosswhite, H. Crosswhite, Phys. Rev. 169, 130 (1968) http://dx.doi.org/10.1103/PhysRev.169.130.

19. J.A. Barnes, B.L. Carroll, At. Data Nucl. Data Tables 2, I-43 (1970) http://dx.doi .org/10.1016/S0092-640X (70) 80002-X.

20. Z. Rudzikas, J. Vizbaraite, R. Karazija, A. Jucys, Lietuovos Fiz. Rinkinys 1, 54 (1964).

21. Z. Rudzikas, J. Vizbaraite, A. Jucys, Lietuovos Fiz. Rinkinys 1, 37 (1965).

22. R.E. Trees, Phys. Rev. 84, 1089 (1951) http://dx.doi.org/10.1103/PhysRev.84.1089.

23. K. Rajnak, B.G. Wybourne, Phys. Rev. 132, 280 (1963) http://dx.doi.org/10.1103/PhysRev.132.280.

24. K. Rajnak, B.G. Wybourne, Phys. Rev. 134, 596 (1964) http://dx.doi.org/10.1103/PhysRev.134.A596.

25. B.R. Judd, Phys. Rev. 141, 4 (1966) http://dx.doi.org/10.1103/PhysRev.141.4.

26. G. Racah, J. Stein, Phys. Rev. 156, 58 (1967) http://dx.doi.org/10.1103/PhysRev.156.58.

27. S. Feneuille, J. Phys. 28, 61 (1967) http://dx.doi.org/10.1051/jphys:0196700280106100.

28. S. Feneuille, J. Phys. 28, 315 (1967) http://dx.doi.org/10.1051/jphys:01967002803-4031500.

29. S. Feneuille, J. Phys. 28, 497 (1967) http://dx.doi.org/10.1051/jphys:01967002805-6049700.

30. S. Feneuille, J. Phys. 28, 701 (1967) http://dx.doi.org/10.1051/jphys:01967002808-9070100.

31. J. Dembczyński, M. Elantkowska, B. Furmann, J. Ruczkowski, D. Stefańska, J. Phys. B: At. Mol. Opt. Phys. 43, 065001 (2010) http://dx.doi.org/10.1088/0953-4075/43/6/065001.

32. A.P. Yutsis, I.B. Levinson, V.V. Vanagas, Mathematical Apparatus of the Angular Momentum Theory (Vilnius, 1960) (English translation Israel Program for Scientific Translations, Jerusalem, 1962; Gordon and Breach, New York, 1963).

33. C. Schwartz, Phys. Rev. 97, 380 (1955) http://dx.doi.org/10.1103/PhysRev.97.380.

34. C. Schwartz, Phys. Rev. 105, 173 (1955) http://dx.doi.org/10.1103/PhysRev.105.175.

35. P.G.H. Sandars, J. Beck, Proc. R. Soc. A 289, 97 (1965) http://dx.doi.org/10.1098/rspa.1965.0251.

36. J.C. Slater, Phys. Rev. 34, 1293 (1929) http://dx.doi.org/10.1103/PhysRev.34.1293.

37. E.U. Condon, Phys. Rev. 36, 1121 (1930) http://dx.doi.org/10.1103/PhysRev.36.1121.

38. E.U. Condon, G.H. Shortley, The Theory of Atomic Spectra (Cambridge University Press, New York, 1935).

39. B.R. Judd, Rep. Prog. Phys. 48, 907 (1985) http://dx.doi.org/10.1088/0034-4885/48/7/001.

40. A. Pasternak, Z.B. Goldschmidt, Phys. Rev. A 1, 55 (1972) http://dx.doi.org/10.1103/PhysRevA.6.55.

41. B.R. Judd, Proc. R. Soc. 82, 874 (1963) http://dx.doi.org/10.1088/0370-1328/82/6/305.

42. B.R. Judd, La Structure Hyperfine Magnetique des Atomes et des Molecules, edited by R. Lefebvre, C. Moser (C.N.R.S., Paris, 1967) p. 311.

43. P.G.H. Sandars, Adv. Chem. Phys. 14, 365 (1969) http://dx.doi.org/10.1002/9780470143599.ch11.

44. S. Büttgenbach, Hyperfine Structure in 4d-and 5d-Shell Atoms (Springer, Berlin, 1982).

45. G. Olsson, A. Rosen, Phys. Scr. 26, 168 (1982) http://dx.doi.org/10.1088/0031-8949/26/3/006.

46. C. Bauche-Arnoult, Proc. R. Soc. A 322, 361 (1971) http://dx.doi.org/10.1098/rspa.1971.0073.

47. C. Bauche-Arnoult, J. Phys. 34, 301 (1973) http://dx.doi.org/10.1051/jphys:01973003404030100.

48. J. Dembczyński, W. Ertmer, U. Johann, P. Unkel, Z. Phys. A 321, 1 (1985) http://dx.doi.org/10.1007/BF01411937.

49. J. Dembczyński, Physica C 141, 219 (1986) http://dx.doi.org/10.1016/0378-4363(86)90276-7.

50. W. Ertmer, U. Johann, J. Dembczyński, Z. Michalski, Z. Phys. D 2, 67 (1986) http://dx.doi.org/10.1007/BF01437244.

51. R. Aydin et al., Z. Phys. D 15, 281 (1990) http://dx.doi.org/10.1007/BF01437170.

52. J. Dembczyński, G.H. Guthoehrlein, E. Stachowska, Phys. Rev. A 48, 2752 (1993) http://dx.doi.org/10.1103/PhysRevA.48.2752.

53. J. Dembczyński, Phys. Scr. T 65, 88 (1996) http://dx.doi.org/10.1088/0031-8949/1996/T65/012.

54. J. Dembczyński et al., Hyperfine Interact. 127, 49 (2000) http://dx.doi.org/10.1023/A:1012694123192.

55. H.B.G. Casimir, On the Interaction between Atomic Nuclei and Electrons (Teyler's Tweede, Genootshap, 1936).

56. A. Jarosz et al., J. Phys. B: At. Mol. Opt. Phys. 40, 2785 (2007) http://dx.doi.org/10.1088/0953-4075/40/13/019. 